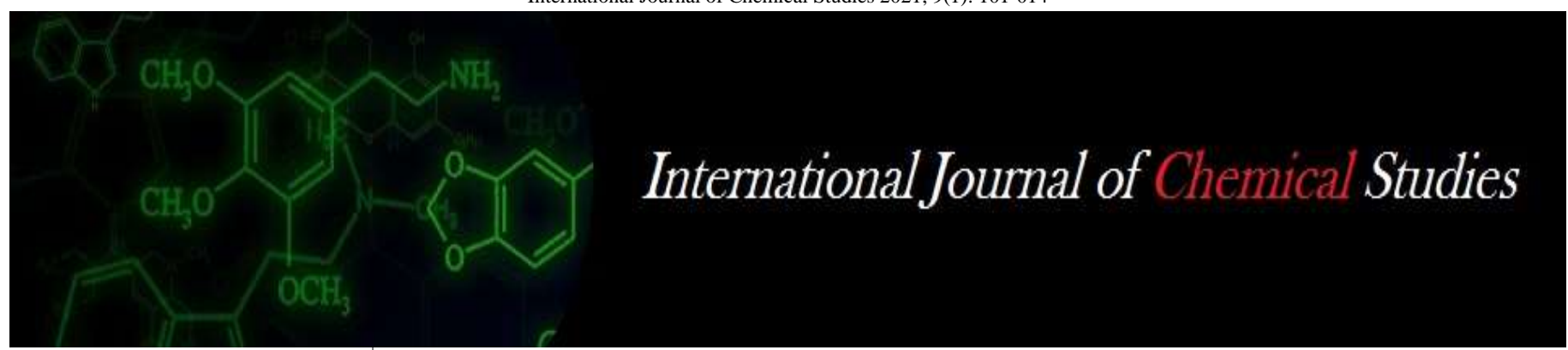

P-ISSN: 2349-8528

E-ISSN: 2321-4902

www.chemijournal.com

IJCS 2021; 9(1): 101-104

(C) 2021 IJCS

Received: 22-10-2020

Accepted: 03-12-2020

SN Kohakade

PGI, MPKV, Rahuri,

Maharashtra, India

Dr. NS Kute

PGI, MPKV, Rahuri,

Maharashtra, India

SH Karvar

PGI, Dr. PDKV, Akola,

Maharashtra, India

Dr. GC Shinde

PGI, MPKV, Rahuri,

Maharashtra, India

\section{Estimation of combining ability effect in mungbean (Vigna radiata (L.) wilczek)}

\author{
SN Kohakade, Dr. NS Kute, SH Karvar and Dr. GC Shinde
}

DOI: https://doi.org/10.22271/chemi.2021.v9.i1b.11211

\begin{abstract}
The present investigation entitled "Estimation of combining ability effect in mungbean (Vigna radiata (L.) Wilczek) was carried out at Post Graduate Farm, MPKV, Rahuri. The material for the present investigation comprised seven parents were crossed in Half diallel fashion to estimate the combining ability for yield and yield attributing traits in mungbean. Analysis of variance revealed significant differences among genotypes, crosses for most of the traits. Preponderance of non-additive gene effects was realized from higher values of specific combining ability compared to general combining ability and ratio of variances of SCA to GCA. The parents showed high GCA can be used for the future hybridization programs. The gca estimates of genotypes emphasized the importance of lines Phule-M402-1, BPMR-145, Utkarsha for their use as a desirable parents for enhancing the yield potential through assembling the favorable genes for yield and yield components. The crosses which showed high SCA effect could be used for the hybrid development. The high yielding crosses viz., Utkarsha $\mathrm{x}$ TM-96-2, Phule-M-605-21 x TMB-146, Phule-M-605-21 x Utkarsha and Phule-M-402-1 x TMB-146 were found to be the superior for seed yield and yield component and should be further tested across the different environment for their stability performance.
\end{abstract}

Keywords: Mungbean, GCA, SCA, diallele

\section{Introduction}

Greengram [Vigna radiata $(\mathrm{L}) \mathrm{R}$. Wilczek] is a short duration legume crop cultivated primarily for its dry seed in tropical, subtropical and temperate zone of Asia including India, Bangaladesh, Pakistan, Myanmar, Indonesia, Philippines, Srilanka, Nepal, China, Korea and Japan (Shanmugasundaram, 2001). However, its yield is much lower than that of other legume crops such as grasspea, chickpea and lentil (FAO, 2007). India is the leading greengram cultivator, with about $55 \%$ of the total world acreage and $45 \%$ of total production (Singh et. al., 2013). According to the report of AICRP MULLaRP (2016), the annual greengram production in India was around 1.5 million tonnes from about 3.02 million ha area during the year 2014-15. Thus, there is an urgent need to increase production and productivity for food and nutritional security which requires efforts to enhance genetic yield potential of the existing varieties by restructuring their plant type. In order to accomplish this task, combining ability analysis was performed which furnishes information to identify desirable parents and genetic architecture of the crosses. It also provides information about the nature of gene action and relative magnitude of fixable and non-fixable genetic variances, which can be used in selecting superior parents and hybrids for production of superior in breds having desirable character(s). It also furnishes the information on additive and non-additive portions of genetic variances present in material for the character under study. Several workers have used combining ability analysis in different crops to study gene effect and genetic worth of parents. But the information on greengram is scanty. Hence the half diallel analysis was adopted in present study to gather information on GCA (general combining abilities) and SCA (specific combining abilities) of parents and simultaneously estimating various types of gene effects involved in the expression of seed yield and related attributes in greengram.

\section{Materials and Methods}

The parent for experiment included seven genotypes of mungbean (Vigna radiate L. Wilczek) were crossed in Diallel mating system at Post Graduate Farm, MPKV, Rahuri. 
All the genotypes ( 7 parents and $21 \mathrm{~F}_{1}$ ) were evaluated in Randomized Block Design with three replication during kharif, 2018. Each genotype was grown in one row of three meter length with a spacing of $30 \mathrm{~cm}$ between row and $10 \mathrm{~cm}$ between plants. Recommended agronomic and plantprotection package of practice were followed to raise healthy crop. Data were recorded on five randomly selected competitive plants in each genotype and replication. Mean value on per plant basis were recorded for the characters, viz., Days to $50 \%$ flowering, Days to maturity, Plant height $(\mathrm{cm})$, Number of clusters per plant, Number of pods per cluster, Number of pods per plants, Pod length $(\mathrm{cm})$, Number of seeds per pod, 100 seed weight (g), Seed yield per plant $(\mathrm{g})$.

The mean data were subjected to statistical analysis. Analysis of variance for combining ability was carried out on the parental and $F_{1}$ means following the experimental method-I, model II of Griffing (1956) ${ }^{[4]}$. The components of variance due to general combining ability $(\sigma 2 \mathrm{gca})$ and due to specific combining ability $(\sigma 2 \mathrm{sca})$ were estimated from the observed and expectations of mean squares under model- II to examine the relative roles of GCA and SCA and hence the nature of gene action. In a half dialed cross, the total genetic variance of the progeny families is given by $2 \sigma 2 \mathrm{gca}+\sigma 2 \mathrm{sca}$ and the phenotypic variance is given by $2 \sigma 2 \mathrm{gca}+\sigma 2 \mathrm{sca}+\sigma 2 \mathrm{e}$.

\section{Results and Discussion}

The analysis of variance for combining ability for the eleven characters revealed highly significant differences in GCA effects and which mean that the parent varieties differ in their general combining ability for these characters and highly significant differences in specific combining ability of the crosses for all characters indicating the importance of both additive and non-additive genetic components of variation in expression of these characters (Table 1). Cheralu et al. (1999), Pandiyan (2006), Jena (2008) ${ }^{[3]}$ and Yadav and Lavanya (2011) had reported similar finding.

Based on the study per se performance of parents and estimates of gca effect parents, Phule-M-402-1 was found to be a good combiner and exhibited significant GCA effects for all the traits excepting number of pods per cluster and pod length. TMB-146 and TM-96-2 was good general combiner for $50 \%$ flowering and days to maturity, while BPMR-145 exhibit significant GCA effect for plant height, pod length, number of seeds per pod and 100 seed weight. Parent Utkarsha was good general combiner for 50\% flowering, days to maturity and yield per plant. Similar results were reported by Jahagirdar (2001) ${ }^{[5]}$, Aher et al., (1999) ${ }^{[1]}$, Singh (2005),
Barad et al., (2008) [3], Patil et al., (2011) and Surashe et al., (2017).

The cross combination Utkarsha $\mathrm{x}$ TM-96-2 recorded highest significant desirable SCA effect for yield per plant (4.238) and number of pods per plant (9.206). Similar result has also been reported by Barad et al., (2008) [3], Patil et al., (2011) and Ahuja (1980) [2], Shanthipriya et al., (2012). This combination also observed highest significant desirable SCA effect for number of seed per pod. These results are in confirmation with the previous work done by Jahagirdar (2001) ${ }^{[5]}$, and Singh and Dikshit (2003).

The highest significant negative desirable SCA effect was observed for days to $50 \%$ flowering and days to maturity in Utkarsha x BPMR-145 similar results were also reported by Jahagirdar (2001) ${ }^{[5]}$.

For plant height in BM-2003-2 x TMB-146 (4.096) was observed highest significant desirable SCA effect. This result was in agreement with the findings of Manjare (1976) ${ }^{[9]}$, Shanthipriya et al., (2012).

The cross combination Phule-M-605-21 x TMB-146 (3.146) had recorded highest significant desirable SCA effect for number of clusters per plant. Similar results were also reported by Manjare (1976) [9], Shanthipriya et al., (2012).

The parents showed high GCA can be used for the future hybridization programmes. The gca estimates of genotypes emphasized the importance of Phule-M-402-1, BPMR-145 and Utkarsha for their use as a desirable parents for enhancing the yield potential through assembling the favourable genes for yield and yield components.

The crosses which showed high SCA effect could be used for the hybrid development. The high yielding crosses viz., Utkarsha x TM-96-2, Phule-M-605-21 x TMB-146, Phule-M605-21 x Utkarsha and Phule-M-402-1 x TMB-146 were found to be the superior for seed yield and yield component and should be further tested across the different environment for their stability performance. Phule-M-402-1 was best combiner for seed yield per plant and other some character like plant height, number of primary branches, number of clusters per plant, number of pod per plant and 100 seed weight, whereas, BPMR 145, plant height, number of seeds per pod, pod length 100 seed weight and TMB-146 for days to $50 \%$ flowering, days to maturity, number of pods per cluster and Utkarsha, pod length, 100 seed weight, yield per plant. Since high gca effect are due to additive and additive $\mathrm{x}$ additive gene action they can be readily exploited in breeding program (Griffing, 1956) ${ }^{[4]}$.

Table 1: Analysis of variance for eleven characters in $7 \times 7$ half diallel crosses in mung bean

\begin{tabular}{|c|c|c|c|c|c|c|c|c|c|c|c|c|}
\hline \multicolumn{13}{|c|}{ Mean sum of squares } \\
\hline $\begin{array}{l}\text { Source of } \\
\text { variation }\end{array}$ & d. f. & $\begin{array}{c}\text { Days to } 50 \% \\
\text { flowering }\end{array}$ & $\begin{array}{c}\text { Days to } \\
\text { maturity }\end{array}$ & $\begin{array}{l}\text { Plant } \\
\text { height }\end{array}$ & $\begin{array}{c}\text { No. of } \\
\text { primary } \\
\text { branches }\end{array}$ & $\begin{array}{c}\text { No. of } \\
\text { clusters per } \\
\text { plant }\end{array}$ & $\begin{array}{l}\text { No. of pods } \\
\text { per clusters }\end{array}$ & $\begin{array}{c}\text { No. of pods } \\
\text { per plant }\end{array}$ & $\begin{array}{c}\text { Pod } \\
\text { length }\end{array}$ & \begin{tabular}{|c|} 
No. of \\
seeds per \\
pod
\end{tabular} & $\begin{array}{c}100 \text { seed } \\
\text { wt. }\end{array}$ & $\begin{array}{c}\text { Grain yield } \\
\text { per plant }\end{array}$ \\
\hline Replication & 2 & 0.32 & 0.04 & 1.25 & 0.00 & 1.55 & 0.02 & 0.52 & 0.02 & 0.07 & 0.02 & 0.17 \\
\hline Treatments & 27 & $36.17 * *$ & $45.19 * *$ & $126.15^{* * *}$ & $0.11 * *$ & $8.69 * *$ & $0.21 * *$ & $61.5 * *$ & $1.58 * *$ & $1.90 * *$ & $1.01 * *$ & $10.62 * *$ \\
\hline Parents & 6 & $46.71 * *$ & $66.04 * *$ & $359.33 * *$ & 0.052 & $5.71 * *$ & $0.14 * *$ & $59.18^{* * *}$ & $3.11 * *$ & $0.84 * *$ & $1.69 * *$ & $10.39 * *$ \\
\hline Hybrids & 20 & $34.23 * *$ & $40.94 * *$ & $39.15 * *$ & $0.087 * *$ & $7.85 * *$ & $0.24 * *$ & $55.33^{* * *}$ & $1.20 * *$ & $2.11 * *$ & $0.83 * *$ & $9.605^{* *}$ \\
\hline $\begin{array}{c}\text { Parent Vs. } \\
\text { Hybrid }\end{array}$ & 1 & $11.57 *$ & $5.14 * *$ & $467.13 * *$ & $1.067 * *$ & $43.43 * *$ & $0.10 *$ & $198.7 * *$ & 0.017 & $4.00 * *$ & $0.46^{* *}$ & $32.41 * *$ \\
\hline Error & 54 & 2.14 & 0.52 & 0.96 & 0.026 & 1.67 & 0.02 & 5.90 & 0.082 & 0.09 & 0.01 & 0.96 \\
\hline Total & 83 & 13.12 & 15.04 & 41.70 & 0.054 & 3.95 & 0.08 & 23.85 & 0.569 & 0.68 & 0.34 & 4.08 \\
\hline
\end{tabular}

*** Significant at 5 and 1 per cent level, respectively 
Table 2: Analysis of variance for combining ability for eleven characters in $7 \times 7$ half diallel crosses in mung bean

\begin{tabular}{|c|c|c|c|c|c|c|c|c|c|c|c|c|}
\hline & & \multicolumn{11}{|c|}{ Mean sum of squares } \\
\hline $\begin{array}{l}\text { Source of } \\
\text { variation }\end{array}$ & d. f. & $\begin{array}{c}\text { Days to 50\% } \\
\text { flowering }\end{array}$ & $\begin{array}{c}\text { Days to } \\
\text { maturity }\end{array}$ & $\begin{array}{c}\text { Plant } \\
\text { height }\end{array}$ & $\begin{array}{c}\text { No. of } \\
\text { primary } \\
\text { branches }\end{array}$ & $\begin{array}{c}\text { No. of } \\
\text { clusters per } \\
\text { plant }\end{array}$ & $\begin{array}{l}\text { No. of pods } \\
\text { per clusters }\end{array}$ & $\begin{array}{c}\text { No. of pods } \\
\text { per plant }\end{array}$ & $\begin{array}{c}\text { Pod } \\
\text { length }\end{array}$ & $\begin{array}{c}\text { No. of } \\
\text { seeds per } \\
\text { pod }\end{array}$ & $\begin{array}{c}100 \text { seed } \\
\text { wt. }\end{array}$ & $\begin{array}{c}\text { Grain } \\
\text { yield per } \\
\text { plant }\end{array}$ \\
\hline GCA & 6 & $36.11 * *$ & $40.96^{* *}$ & $113.85 * *$ & $0.02 *$ & $3.22 * *$ & $0.04 * *$ & $15.32 * *$ & $1.36^{* *}$ & $0.52 * *$ & $1.08 * *$ & $2.11 * *$ \\
\hline SCA & 21 & $5.182 * *$ & $7.66^{* *}$ & $21.53 * *$ & $0.04 * *$ & $2.80 * *$ & $0.08 * *$ & $21.98^{* * *}$ & $0.28 * *$ & $0.66 * *$ & $0.12 * *$ & $3.95 * *$ \\
\hline Error & 54 & 0.71 & 0.17 & 0.32 & 0.009 & 0.55 & 0.008 & 1.96 & 0.027 & 0.033 & 0.005 & 0.32 \\
\hline$\sigma^{2}$ gca & & 3.93 & 4.53 & 12.61 & 0.001 & 0.29 & 0.003 & 1.48 & 0.14 & 0.05 & 0.11 & 0.19 \\
\hline$\sigma^{2} \mathrm{sca}$ & & 4.46 & 7.48 & 21.21 & 0.03 & 2.24 & 0.071 & 20.01 & 0.26 & 0.63 & 0.12 & 3.2 \\
\hline$\sigma^{2} \mathrm{gca} / \sigma^{2} \mathrm{sca}$ & & 0.88 & 0.60 & 0.59 & 0.03 & 0.13 & 0.05 & 0.07 & 0.56 & 0.08 & 0.99 & 0.05 \\
\hline
\end{tabular}

*** Significant at 5 and 1 per cent level, respectively

Table 3: Estimates of general combining ability effects of parents for eleven characters in mung bean

\begin{tabular}{|c|c|c|c|c|c|c|c|c|c|c|c|c|}
\hline $\begin{array}{l}\text { Sr. } \\
\text { No. }\end{array}$ & Parents & $\begin{array}{c}\text { Days to } 50 \% \\
\text { flowering }\end{array}$ & $\begin{array}{c}\text { Days to } \\
\text { maturity }\end{array}$ & $\begin{array}{l}\text { Plant } \\
\text { height }\end{array}$ & $\begin{array}{c}\text { No. of } \\
\text { primary } \\
\text { branches }\end{array}$ & $\begin{array}{c}\text { No. of } \\
\text { clusters per } \\
\text { plant }\end{array}$ & $\begin{array}{l}\text { No. of pods } \\
\text { per clusters }\end{array}$ & $\begin{array}{c}\text { No. of pods } \\
\text { per plant }\end{array}$ & \begin{tabular}{c|} 
Pod \\
length
\end{tabular} & \begin{tabular}{|c|} 
No. of \\
seeds per \\
pod
\end{tabular} & $\begin{array}{c}100 \text { seed } \\
\text { wt. }\end{array}$ & \begin{tabular}{|c|} 
Grain \\
yield per \\
plant
\end{tabular} \\
\hline 1 & Phule-M-605-21 & $3.34 * *$ & $2.97 * *$ & $3.98 * *$ & 0.043 & $0.54 *$ & -0.04 & 0.65 & $\mid-0.44 * *$ & -0.20 ** & $-0.24 * *$ & \begin{tabular}{|l|}
-0.21 \\
\end{tabular} \\
\hline 2 & Phule-M-402-1 & $1.01 * *$ & $1.83 * *$ & $1.46 * *$ & $0.073 *$ & $0.73 * *$ & 0.01 & $2.27 * *$ & \begin{tabular}{|l|}
-0.06 \\
\end{tabular} & $-0.15 * *$ & $0.16 * *$ & $0.78 * *$ \\
\hline 3 & Utkarsha & $-0.68 *$ & $-1.13 * *$ & $-1.53 * *$ & -0.016 & -0.14 & 0.03 & -0.36 & $0.28 * *$ & -0.02 & $0.35 * *$ & $0.46 *$ \\
\hline 4 & BM-2003-2 & 0.095 & $-0.57 * *$ & $-1.47 * *$ & -0.038 & -0.03 & 0.003 & -0.73 & $0.42 * *$ & $0.12 *$ & $0.13 * *$ & 0.07 \\
\hline 5 & BPMR-145 & $0.90 * *$ & $1.49 * *$ & $4.14 * *$ & 0.029 & $-0.67 * *$ & $-0.08 * *$ & $-1.95^{* *}$ & $0.47 * *$ & $0.47 * *$ & $0.39 * *$ & -0.19 \\
\hline 6 & TM-96-2 & $-1.76^{* *}$ & $-1.72 * *$ & $-0.59 * *$ & -0.038 & 0.39 & -0.052 & 0.29 & $-0.32 * *$ & $-0.20 * *$ & $-0.33^{* *}$ & -0.26 \\
\hline \multirow[t]{4}{*}{7} & TMB-146 & $-2.75 * *$ & $-2.87 * *$ & $-5.98 * *$ & -0.053 & $-0.82 * *$ & $0.12 * *$ & -0.16 & $-0.34 * *$ & -0.023 & $-0.47 * *$ & $-0.64 * *$ \\
\hline & $\mathrm{SE}(\mathrm{i})$ & 0.261 & 0.129 & 0.175 & 0.028 & 0.230 & 0.027 & 0.432 & 0.050 & 0.056 & 0.022 & 0.174 \\
\hline & $\mathrm{CD}$ at $5 \%$ & 0.523 & 0.259 & 0.351 & 0.057 & 0.461 & 0.054 & 0.867 & 0.102 & 0.112 & 0.044 & 0.349 \\
\hline & $\mathrm{CD}$ at $1 \%$ & 0.68 & 0.340 & 0.462 & 0.073 & 0.607 & 0.071 & 1.140 & 0.132 & 0.147 & 0.058 & 0.459 \\
\hline
\end{tabular}

*** Significant at 5 and 1 per cent level, respectively

Table 4: Estimates of specific combining ability effects of crosses for eleven characters in mung bean

\begin{tabular}{|c|c|c|c|c|c|c|c|}
\hline $\begin{array}{l}\text { Sr. } \\
\text { No. }\end{array}$ & Crosses & $\begin{array}{c}\text { Days to } 50 \% \\
\text { flowering }\end{array}$ & $\begin{array}{c}\text { Days to } \\
\text { maturity }\end{array}$ & Plant height & $\begin{array}{c}\text { No. of primary } \\
\text { branches }\end{array}$ & $\begin{array}{c}\text { No. of clusters } \\
\text { per plant }\end{array}$ & $\begin{array}{l}\text { No. of pods } \\
\text { per clusters }\end{array}$ \\
\hline 1 & Phule-M-605-21 X Phule-M-402-1 & -0.389 & $2.333 * *$ & $-6.693 * *$ & -0.093 & -0.413 & $-0.470 * *$ \\
\hline 2 & Phule-M-605-21 X Utkarsha & $1.981 *$ & $2.296^{* *}$ & $1.782^{* *}$ & 0.063 & $-1.458^{*}$ & $-0.412 * *$ \\
\hline 3 & Phule-M-605-21 X BM-2003-2 & $-2.278 * *$ & $-2.259 * *$ & $-7.444 * *$ & 0.019 & 0.032 & $0.411 * *$ \\
\hline 4 & Phule-M-605-21 x BPMR-145 & 0.722 & $-1.333 * *$ & $-1.158^{*}$ & $0.219 * *$ & 0.998 & -0.063 \\
\hline 5 & Phule-M-605-21 x TM-96-2 & $3.056^{* *}$ & $1.556^{* *}$ & 0.179 & 0.152 & -0.273 & -0.115 \\
\hline 6 & Phule-M-605-21 x TMB-146 & $2.352 * *$ & $1.037 * *$ & $1.139 *$ & 0.033 & $3.146 * *$ & $0.196^{*}$ \\
\hline 7 & Phule-M-402-1 X Utkarsha & -1.352 & $-2.889 * *$ & 0.971 & -0.100 & -0.942 & $0.324 * *$ \\
\hline 8 & Phule-M-402-1 x BM-2003-2 & $2.389^{* *}$ & $4.889 * *$ & $-4.489 * *$ & 0.122 & -1.188 & 0.027 \\
\hline 9 & Phule-M-402-1 x BPMR-145 & $3.056^{* *}$ & 0.481 & $-3.802 * *$ & $0.189^{*}$ & $2.269 * *$ & -0.120 \\
\hline 10 & Phule-M-402-1 x TM-96-2 & -1.278 & 0.037 & $-2.032 * *$ & -0.078 & $-1.198 * *$ & $-0.285 * *$ \\
\hline 11 & Phule-M-402-1 x TMB-146 & $-2.315^{* *}$ & $-1.148^{* *}$ & 0.494 & $0.337 * *$ & $2.664 * *$ & -0.035 \\
\hline 12 & Utkarsha x BM-2003-2 & $2.093 * *$ & -0.481 & $-2.594 * *$ & -0.056 & -0.250 & $-0.169 *$ \\
\hline 13 & Utkarsha x BPMR-145 & $-2.907 * *$ & $-3.556^{* *}$ & $-6.107 * *$ & 0.078 & -0.543 & 0.118 \\
\hline 14 & Utkarsha x TM-96-2 & -0.574 & $1.667 * *$ & 0.029 & $0.344 * *$ & 0.957 & $0.499 * *$ \\
\hline 15 & Utkarsha x TMB-146 & -1.27 & $-1.852 * *$ & $1.456 * *$ & $-0.174 *$ & $2.926 * *$ & $-0.470 * *$ \\
\hline 16 & BM-2003-2x BPMR-145 & $-1.500^{*}$ & $-0.778^{*}$ & 0.167 & $0.167 *$ & -0.495 & -0.139 \\
\hline 17 & BM-2003-2 x TM-96-2 & -0.833 & $-3.556^{* *}$ & -0.030 & 0.100 & -4.20 & 0.049 \\
\hline 18 & BM-2003-2x TMB-146 & $-2.204 * *$ & $-1.741 * *$ & $4.096 * *$ & 0.115 & 0.442 & $0.233 * *$ \\
\hline 19 & BPMR-145 x TM-96-2 & -0.833 & $-1.630 * *$ & $-5.077 * *$ & $-0.300 * *$ & $1.795 * *$ & -0.011 \\
\hline 20 & BPMR-145 x TMB-146 & $3.130 * *$ & $-1.481 * *$ & 0.136 & -0.019 & 0.409 & -0.134 \\
\hline \multirow[t]{4}{*}{21} & TM-96-2 х TMB-146 & $3.463 * *$ & $5.407 * *$ & 0.386 & $0.248 * *$ & -0.443 & 0.148 \\
\hline & SE(i) & 0.759 & 0.376 & 0.509 & 0.083 & 0.670 & 0.079 \\
\hline & CD at $5 \%$ & 1.522 & 0.755 & 1.022 & 0.166 & 1.343 & 0.159 \\
\hline & $\mathrm{CD}$ at $1 \%$ & 2.003 & 0.995 & 1.346 & 0.219 & 1.768 & 0.208 \\
\hline
\end{tabular}

*** Significant at 5 and 1 per cent level, respectively

\begin{tabular}{|c|c|c|c|c|c|c|}
\hline Sr. No. & Crosses & No. of pods per plant & Pod length & No. of seeds per pod & 100 seed wt. & Grain yield per plant \\
\hline 1 & Phule-M-605-21 X Phule-M-402-1 & $-6.532^{* *}$ & -0.036 & $0.796^{* *}$ & $-0.225^{* *}$ & 0.885 \\
\hline 2 & Phule-M-605-21 X Utkarsha & $-3.891^{* *}$ & 0.140 & -0.239 & $-0.366^{* *}$ & $2.890^{* *}$ \\
\hline 3 & Phule-M-605-21 X BM-2003-2 & 1.672 & $-1.056^{* *}$ & $-0.407^{* *}$ & $-0.633^{* *}$ & $-1.194^{* *}$ \\
\hline 4 & Phule-M-605-21 x BPMR-145 & $0.498^{* *}$ & -0.167 & 0.181 & 0.121 & 0.164 \\
\hline 5 & Phule-M-605-21 x TM-96-2 & -1.154 & 0.178 & -0.090 & $0.777^{* *}$ & 0.041 \\
\hline 6 & Phule-M-605-21 x TMB-146 & $5.239^{* *}$ & $0.618^{* *}$ & $0.321^{*}$ & $0.161^{*}$ & $3.758^{* *}$ \\
\hline 7 & Phule-M-402-1 X Utkarsha & 0.427 & -0.205 & -1.656 & 0.004 & 0.305 \\
\hline 8 & Phule-M-402-1 x BM-2003-2 & 1.596 & $-0.324^{*}$ & -0.804 & -0.016 & $1.338^{* *}$ \\
\hline 9 & Phule-M-402-1 x BPMR-145 & $-3.317^{* *}$ & $1.052^{* *}$ & $0.510^{* *}$ & -0.055 & $-1.524^{* *}$ \\
\hline 10 & Phule-M-402-1 x TM-96-2 & 2.364 & $-0.520^{* *}$ & 0.040 & $0.237^{* *}$ & $1.206^{* *}$ \\
\hline 11 & Phule-M-402-1 x TMB-146 & $7.490^{* *}$ & 0.227 & $0.517^{* *}$ & $0.417^{* *}$ & $2.026^{* *}$ \\
\hline 12 & Utkarsha x BM-2003-2 & -1.269 & $-0.798^{* *}$ & $0.695^{* *}$ & $0.369^{* *}$ & $-1.171^{* *}$ \\
\hline
\end{tabular}




\begin{tabular}{|c|c|c|c|c|c|c|}
\hline 13 & Utkarsha x BPMR-145 & $3.991 * *$ & $-0.589 * *$ & $-0.490 * *$ & 0.083 & $1.368 * *$ \\
\hline 14 & Utkarsha x TM-96-2 & $9.206 * *$ & 0.183 & $1.699 * *$ & 0.052 & $4.238 * *$ \\
\hline 15 & Utkarsha x TMB-146 & $-4.402 * *$ & -0.154 & $-0.444 * *$ & $0.186 * *$ & $-1.609 * *$ \\
\hline 16 & BM-2003-2x BPMR-145 & -0.714 & $0.599 * *$ & $0.861 * *$ & -0.063 & 0.907 \\
\hline 17 & BM-2003-2 x TM-96-2 & 0.101 & $0.607 * *$ & $-0.610 * *$ & $-0.168 *$ & 0.057 \\
\hline 18 & BM-2003-2x TMB-146 & $6.894 * *$ & $0.607 * *$ & $0.774 * *$ & $-0.148 *$ & $1.831 * *$ \\
\hline 19 & BPMR-145 x TM-96-2 & 1.528 & -0.074 & -0.008 & $0.236 * *$ & 0.809 \\
\hline 20 & BPMR-145 x TMB-146 & -2.079 & -0.074 & $0.916 * *$ & $0.410 * *$ & $-1.228 * *$ \\
\hline \multirow[t]{4}{*}{21} & TM-96-2 x TMB-146 & 1.002 & 0.138 & 0.085 & $-0.478 * *$ & -0.015 \\
\hline & SE(i) & 1.258 & 0.148 & 0.163 & 0.064 & 0.507 \\
\hline & $\mathrm{CD}$ at $5 \%$ & 2.523 & 0.296 & 0.326 & 0.129 & 1.017 \\
\hline & $\mathrm{CD}$ at $1 \%$ & 3.321 & 0.390 & 0.430 & 0.170 & 1.338 \\
\hline
\end{tabular}

*** Significant at 5 and 1 per cent level, respectively

\section{References}

1. Aher RP, Datur DV, Sonawane YP. Combining ability in mungbean. Crop Res 1999;18:256-260.

2. Ahuja SL. Diallel analysis in F2 generation of mungbean, Thesis Abstract (NAU-Hissar) 1980;6(2):110-111.

3. Barad HR, Pithia MS, Vachhani JH. Heterosis and combining ability studies for economic traits in mungbean (Vigna radiate (L.) Wilczek). Legume Res 2008, P31.

4. Griffing B. Concept of general and specific combining ability in relation to diallel crossing system. Australian J Biol. Sci 1956;9:484-493.

5. Jahagirdar JE. Heterosis and combining ability studies for seed yield and yield components in mungbean. Indian $\mathrm{J}$ pulses Res 2001;14(2):141-142.

6. Katiyar M, Amit Kumar. Genetics Analysis of yield and its component Traits in Mungbean (Vigna radiataL. Wilczek). Int. J Innovative Res. Dev 2015;4(2):119-121.

7. Kempthorne O. An introduction to genetical statistics. John Wiley and Sons, New York 1957.

8. Khattak GSS, Haq MA, Ashraf M, Srinives P. Combining ability in mungbean (Vigna radiata $(L)$ Wilczek) I. Agronomic traits. Korean J Crop Sci 2001;46(5):420-423.

9. Manjare MR. Studies on heterosis and combining ability in $8 \times 8$ diallel cross of mungbean. M. Sc. (Agri.). Thesis Mahatma Phule Krishi Vidyapeeth, Rahuri 1976.

10. Panse VG, Sukhatme PV. Statistical methods for agricultural work. Indian council of Agric. Res., New Delhi 1967, P167-174.

11. Sathya M, Jayamani P. Heterosis and combining ability studies in greengram. $\mathrm{J}$ of Food Legumes 2011;24(4):282-287.

12. Shanthi Priya M, Reddy KHP, Reddy DM, Rupesh Kumar Reddy. Combining ability studies in greengram. (Vigna radiate (L.) 2012. 